\title{
Universal health coverage in Iran: What kind of knowledge is needed to achieve the goals?
}

\author{
Zhaleh Abdi $^{1}$, Bahareh Yazdizadeh*2, Elham Ahmadnezhad ${ }^{1}$, Mahboubeh Rahimi ${ }^{1}$, Reza Majdzadeh ${ }^{3}$
}

Received: 19 Aug 2018

Published: 8 Jul 2019

\begin{abstract}
Achieving universal health coverage (UHC), which means ensuring access to high quality and equitable services by all without financial hardship, requires local evidence. To find interventions appropriate to local needs, local knowledge and evidence are required in addition to global evidence. Thus, every country should have its own plan for research production and utilization and strengthening researchers' capacities to achieve UHC. To accomplish the goals of UHC, the research system should be able to determine the research priorities and agenda, collect resources, improve the capacity for evidence generation, and maximally utilize the country's capacity for finding local solutions by establishing research networks. In this study, inputs for UHC research priority setting in Iran and its challenges have been discussed.
\end{abstract}

Keywords: Universal health coverage, Health system research

Conflicts of Interest: None declared

Funding:None

*This work has been published underCC BY-NC-SA 1.0 license.

Copyright $₫$ Iran University of Medical Sciences

Cite this article as:Abdi Zh, Yazdizadeh B, Ahmadnezhad E, Rahimi M, Majdzadeh R. Universal health coverage in Iran: What kind of knowledge is needed to achieve the goals?.Med J Islam Repub Iran. 2019(8 Jul);33:65.https://doi.org/10.47176/mjiri.33.65

\section{Introduction}

In recent years, strengthening health systems has been put on the agenda with the aim of achieving universal health coverage (UHC). In Iran, multiple interventions have been launched to strengthen the health system and accelerate the attainment of UHC. The most notable step taken in recent years is the Health Transformation Plan (HTP). In spite of commitment towards achieving UHC, there is still a long way till its goals are realized, and it is unclear how access to quality services and financial protection for all and in all settings will be possible.

To attain UHC, 2 sets of questions should be answered by evidence (1). The first group of questions lays emphasis on the necessary interventions: Which services should be delivered? Which level of services (ie, from district level to referral hospital) should be provided? How can we promote financial protection? Also, the know-how of ac-

Corresponding author: Dr Bahareh Yazdizadeh,byazdizadeh@tums.ac.ir

1. National Institute for Health Research, Tehran University of Medical Sciences, Tehran, Iran

2. Knowledge Utilization Research Center, Tehran University of Medical Sciences, Tehran, Iran

3. Community-Based Participatory-Research Center, Knowledge Utilization Research Center, Tehran University of Medical Sciences, Tehran, Iran cess to health care services and protection from financial risk demand local answers. Therefore, in addition to using international evidence, countries need local contextual knowledge to inform the development of strategies aimed at accelerating UHC [2]. The second group of questions arise from the fact that UHC is an approach towards strengthening the health system. However, which topics in UHC goals (eg, diseases) must be dealt with and the extent to which these goals were met still depend on the countries' priorities (2). Thus, local research must answer questions on the extent of progress towards UHC and achieving UHC goals. Knowledge can improve UHC, however, a variety of ways can also improve UHC: feedback from the impact on health-related policies and making adjustments based on the received feedback; improving the access, utilization, and quality of health care deliv-

$\uparrow$ What is "already known” in this topic:

To achieve UHC, some questions must be answered based on contextual evidence. These questions have been clarified in WHO documents, however, the needed evidence must be determined in each country according to its context.

$\rightarrow$ What this article adds:

In this editorial paper, the existing data on sources of the needed evidence for achieving UHC in Iran have been collected and summarized. 
ery by acknowledging the status of each; and finally capacity building of health care providers.

In this study, to find which queries have been answered by the studies conducted in the country, all the articles that had been published in English and Persian journals after the implementation of the HTP up to May 2018 were examined. Most of the studies were concerned with satisfaction evaluation of patients and service providers following HTP, the rate of out-of-pocket payment of households for health services, the extent of households' exposure to catastrophic costs, the extensiveness of informal payments, changes in hospitals' functional indicators, and changes in rates of cesareans and natural births after HTP implementation. This is despite the fact that the following issues have not yet been adequately addressed at local level: the level of access to different services and the changes brought about in them; the effective coverage of services; changes in health status; and the technical quality of services delivered by medical centers.

It seems that research on important topics, such as UHC and HTP, has not attracted enough attention in Iran. The low ratio of health services research (3) and statements made by managers and policymakers on the scarcity of applied research (4) bear witness to this claim. Therefore, given the shortage of resources, particularly, capable researchers, and conducting priority-based research will shorten the time taken to achieve UHC. Accordingly, preparing a list of research priorities for UHC and encouraging researchers to conduct research based on this list is of utmost importance at this time.

(1) Priority setting is an important procedure in the management of health research across countries, and it becomes even more important in light of the shortage of financial and human resources in developing countries like Iran. There are standard methods for setting research priorities. These methods should be employed more often by governments in setting UHC research priorities at national level to ensure maximum utilization of the available resources. To this end, a study was conducted using the Child Health and Nutrition Research Initiative (CHNRI) approach to determine Iran's research priorities for the next 5 years to help the country achieve its long-term health goals. The long-term goals of the study were extracted from 2 documents: (1) National General Health Policies by 2025 and (2) the health targets of the Sustainable Development Goals by 2030 (SDGs). Unlike older methods for setting research priorities, the CHNRI approach has a systematic and transparent approach that helps researchers independently write their research ideas and to score a list of collected ideas. Moreover, it engages researchers, research funders, policymakers, and stakeholders from the primary stages. More than 50 researchers from the health sector participated in this study, and a total of 128 research topics were selected as priorities of the health system. Among the first 10 priorities, 6 were directly related to UHC: health insurance system's reform to improve equity; (2) integration of NCD prevention strategy into primary health-care; (3) identification of costeffective population-level interventions for NCD and road injury prevention; (4) epidemiological assessment of
NCDs in Iran by geographic areas; (5) tracking the equality of distribution of health resources and services across the country; (6) investigating the current and the future common health problems in Iran's elderly and identifying strategies to reduce the consequent economic burden (5).

Another resource that can be used as a guide for research is UHC monitoring and evaluation (M\&E) framework. Some studies must be conducted to ensure the achievement of UHC goals. The HTP monitoring and evaluation design began in June 2014 (almost at the same time as the plan began in the field). The assessment of the reform plan was handed over to the National Institute of Health Research (NIHR), which is responsible for generating evidence for health policymakers. Before that, there was no well-defined M\&E framework to assess progress towards the accomplishment of UHC and/or realizing the goals of the HTP. Given that HTP was implemented as a reform of the health system to facilitate access to UHC, a comprehensive framework was designed to monitor and evaluate HTP and to report UHC achievements at the international level. The M\&E framework was designed using literature review based on the World Bank and World Health Organization's recommended framework (6). The framework formulation process was performed through a series of meetings with experts and senior managers working at the Ministry of Health and Medical Education of Iran (MOHME). The final draft was presented to policymakers for input and approval. The framework indicators fall into 4 main domains (input, output, impact, outcome) and are classified into 12 overall groups (Table 1). These indicators can be extracted from different sources. Some of them are extracted from national surveys, some from routine information systems, and some from cross sectional studies that can be considered as research topics. Since the achievement of equity is implicit in UHC goals, disaggregation of the indicators by the main equity stratifiers was taken into consideration during the $M \& E$ framework development.

Data should be collected routinely for all these indicators. Designing the M\&E framework for the HTP sheds light on the importance of further Investment in the health information system. Investment is required to strengthen the capacity of the health system to generate high quality information for monitoring progress towards UHC. The information gap that hampers the monitoring of the progress towards the UHC should be addressed through regular and periodic surveys that capture all the dimensions of UHC.

\section{UHC research requirements and barriers}

Every country faces a series of barriers and challenges in achieving UHC. The human resources for UHC research are still inadequate in Iran. Institutions working to accelerate progress towards UHC in Iran face multiple barriers and difficulties in conducting and implementing UHC research. The interventions and decisions that can improve UHC do not take place in the MOHME alone. Also, the policies and decisions are made by a number of other organizations and institutions that have direct and fundamental roles in accomplishing the aim too, including 
Table 1. Monitoring and Evaluation Framework of Iran's Health Transformation Pla: Towards Universal Health Coverage

\section{INPUT}

1. Financing

1. Total Health Expenditure (THE) per\% GDP

2. Total Health Budget per\% Government Budget

3. Total Health Expenditure (THE) per capita

4. General Government Health Expenditure per \%GGE (Constant price \& Constant PPPs)

5. Total Pharmaceutical Expenditure per Capita (Constant price \& Constant PPPs)

6. Fair Financial Contribution Index

7. \%Total Health Expenditure (THE) by Type of Financing Agents

8. Out of Pocket Payment, \%THE (Total (and separate) by Pharmaceutical, Dental and Imaging Services)

2. Infrastructure and Health

1. General Physician per 10,000 pop

2. Specialist per 10,000 pop

3. Paramedic per 10,000 pop

4. Nurses per Hospital Bed

5. Dentist per 10,000 pop

6. Hospital Bed per 10,000 pop

3. Information System

1. Number (\%) of Health Facilities has Access to Computer with Email/Internet Access

2. Number (\%) of Hospital connect to SEPAS (the integrated national health information system System)

OUTPUT

4. Access

1. Access to Health Services (Outpatient-Inpatient)/(Public-non-Public-Private) (Pre-Hospital Services

2. Access to Medicines and Medical Devices in Public Hospital

3. Access to Essential Drugs (WHO Suggested List)

5. Coverage

1. Contraceptive Prevalence Rate and Profiles

2. Insurance Coverage (Self-Reported)

3. Receipt of Preventive Services (Pregnancy Care, Diabetes, Hypertension, TB, Immunization and Depression)

4. Coverage of Exclusive Breastfeeding

5. Pentavalent Coverage, $\%$ children $<1$

6. Measles Coverage, $\%$ children $<1$

7. Coverage of Preventive Cardiovascular Care for High Risk Group

8. Skilled Birth Attendance

9. Suspected Pneumonia Treated with Antibiotic

10. Diarrhea Treated with Oral Rehydration Salts (ORS)

11. Condom use at Higher Risk Sex

12. Coverage of Mammography (for 40-65 years old/Biennial)

6. Utilization

1. Outpatient Visit per capita

2. Admission Rate per capita

3. Consumption of Medicine per Capita in Volume-Value (IP/OP)

4. OP/IP use Profile (Public/nonPublic/Private)

5. Unmet Healthcare needs (OP/IP)

7. Safety \& Quality

1. Accredited Hospitals

2. Success TB Treatment Rate

3. Smoking Cessation Rate

4. Case Fatality Rate in Hospital (Acute MI \& Stroke)

5. Waiting Time for Elective Surgery (Cataract-HIP/Knee Replacement)

6. four ANC visits, $\%$ total

7. Asthma (re) Admission Rate

8. Adherence to Treatment by Guidelines-(Angiography, Angioplasty, Laboratory Tests, Cataract and Imaging)

9. Number (\%) of Normal Tests (MRI, Ct-Scan, Angiography, Angioplasty and Laboratory Tests
Available but are not produced routinely Available but are not produced routinely Available but are not produced routinely Available but are not produced routinely Available but are not produced routinely Not available

Not available

Available but needs modification

Available but needs modification Available but needs modification Available but needs modification Available but needs modification Available but needs modification Available but needs modification

Not available

Not available

Available but are not produced routinely Not available

Not available

Available but are not produced routinely Available but are not produced routinely Available but needs modification

Available but are not produced routinely Available but are not produced routinely Available but are not produced routinely Available but are not produced routinely Available but are not produced routinely Available but are not produced routinely Available but are not produced routinely Not available

Not available

Available but are not produced routinely Available but needs modification

Not available

Available but are not produced routinely Available but are not produced routinely

Available but needs modification

Available but needs modification Available but are not produced routinely Not available

Not available

Available but are not produced routinely

Not available

Not available

Not available the Ministry of Welfare and Social Security, the Rehabilitation Organization, the Imam Khomeini Relief Foundation, the Red Crescent Society, and public and private insurance organizations. Therefore, improving the performance of these organizations' research systems has a direct impact on the improvement of evidence-informed policymaking in these organizations. These organizations face a number of barriers in various aspects of their research systems : (1) financing (lack of a budget line for research); (2) producing and using research (lack of a structured and systematic method to identify research pri-

ority, lack of stakeholders engagement in priority setting, lack of qualified researchers, non-existence of a proper knowledge translation platform, lack of active dissemination of research results to stakeholders, absence of a unit responsible for collecting research findings of policymaking research, and lack of a clear plan for translating research findings to practice); (3) creating and sustaining resources (serious shortage of qualified researchers); (4) stewardship (limited direct investment in capacity development for health systems research, absence of a specific structure for integrating research finding into practice at 
Table 1.Ctd

OUTCOME

8. Effective Coverage

1. Angina Treatment Coverage

2. Hypertension Treatment Coverage

3. Diabetes Treatment Coverage

4. Mental Health: Depression Treatment Coverage

5. Asthma/ COPD Treatment Coverage

6. Coverage of Renal Replacement Therapy

7. Arthritis Treatment Coverage

8. Hearing Aid Coverage (Elderly who need a hearing aid)

9. Rapid Care in Hospital for Cardiovascular Disease (CVD)

10. Palliative Care Coverage

11. Cataract Surgical Coverage

12. Dental Care Coverage

13. Cesarean/Section Rate

9. Risk Factors

1. Children under 5 who are Stunted

2. Children under 5 who are Underweight

3. Children under 5 who are Overweight

4. Low Birth Weight

5. Improved Water

6. Improved Sanitation

7. Number of Qualified Air

8. Percentage of the Population that is Overweight and Obese

9. Current Tobacco Smoking

10. Current Non-Tobacco Smoking

11. Age-standardized mean population Intake of Salt (Sodium Chloride) per day in Grams in Persons Aged $18+$

12. Prevalence of persons aged $18+$ ) consuming less than five total Servings (400g) of Fruit and Vegetable per day

IMPACT

10. Health Status

1. Life Expectancy at Birth

2. Maternal Mortality Ratio (100,000 Live Birth)

3. Under-5 Mortality Rate (1000 live birth)

4. Self-Reported Health Status

5. Age-standardized Prevalence of Diabetes (based on HbA1c Levels), Hypertension, Cardiovascular Disease and Chronic Respiratory Disease

6. TB Case Detection Rate (the number of estimated new TB cases detected in a given year using the DOTS approach) Expressed as a Percentage of all new TB Cases

7. 5year Survival Rate (specific cancer) - Breast, Colon, Gastric, Prostate

8. Survival Curve of Specific Diseases: End Stage Renal

9. Patients under Renal Replacement Therapy

10. Suicide Rate

11. Road Traffic Deaths per 100,000

11. Financial Risk Protection(3)

1. Catastrophic Health Expenditure

2. Impoverishment Health Expenditure

12. Satisfaction

1. Patient Satisfaction

2. Population Satisfaction (from Health Services)

3. Provider Satisfaction

1. Available but needs modification

2. Available but are not produced routinely

3. Not available

MOHME, lack of willingness or motivation of policymakers and manages to use evidence in policy, not enough investment in research to implement policies and programs).

The challenges faced at macro level by the HTP are classified in 3 important domains: (1) unsustainable financing, (2) inefficiency of the health system, and (3) weakness of governance. Then, 6 interventions are recognized as the response to aforementioned challenges (8). Table 2 presents these 6 interventions along with the required research questions. A wide range of scientific evidence is necessary to confront the existing difficulties. Under the current circumstances, the greatest need is felt for identifying and defining problems and their determi-
Available but are not produced routinely Available but are not produced routinely Available but are not produced routinely Not available Not available

Not available

Not available

Not available

Not available

Not available

Not available

Not available

It is produced routinely

Not available

Not available

Not available

Available but are not produced routinely Available but are not produced routinely Available but are not produced routinely Not available

Available but are not produced routinely Available but are not produced routinely Available but are not produced routinely Available but are not produced routinely

Available but are not produced routinely

Available

Available but needs modification

It is produced routinely

Available but are not produced routinely Available but needs modification

Available

Not available

Not available

Not available

Not available

Available but needs modification

Available but are not produced routinely

Available but are not produced routinely It is produced routinely

Available but are not produced routinely Available but are not produced routinely Available but are not produced routinely Available but are not produced routinely

\section{$4 \quad$ http://mjiri.iums.ac.ir}

$$
\text { Med J Islam Repub Iran. } 2019 \text { (8 Jul); 33:65. }
$$

nants and identifying their appropriate interventions. In terms of knowledge, a wide range of research is needed to respond to the current questions posed, ranging from theory-based studies for identifying appropriate interventions to interventional studies recommending the most appropriate intervention at the national level. However, a review of the studies conducted after HTP indicate that most studies have concentrated on identifying and defining the problems and have not presented solutions and/or addressed their implementation and assessment.

UHC research will be effective only when and if it is conducted within a supportive national research system. Such a system has many important applications: (1) it should be able to determine the research priorities in ac- 
Table 2.Interventions proposed for addressing the reform plan's challenges and relevant questions

Table 2: Interventions proposed for addressing the reform plan's challenges and relevant questions

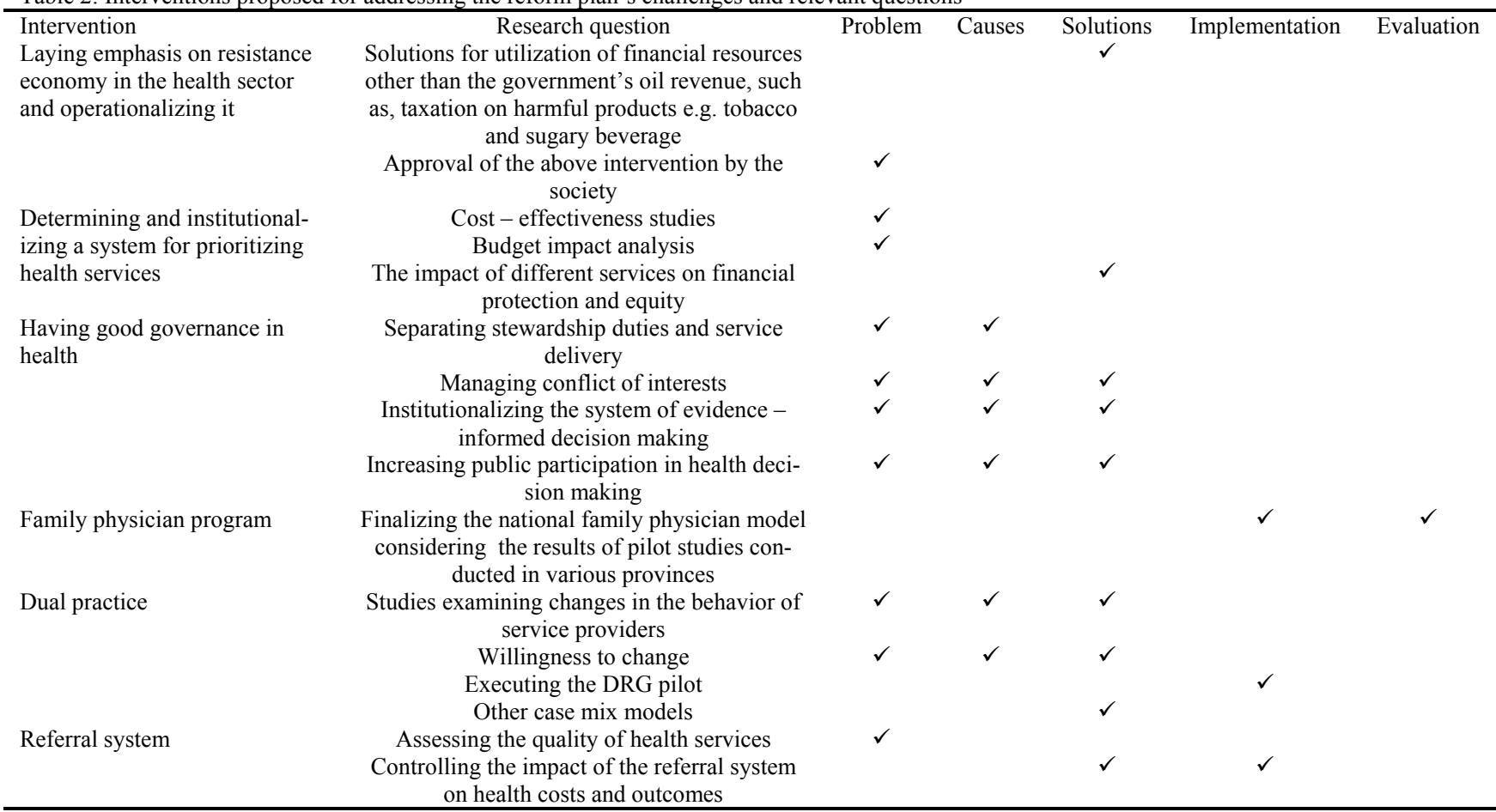
on health costs and outcomes

cordance with the context; (2) it should be able to create the necessary capacity for research at national level; (3) it should be able to determine the required standards and norms for research; (4) eventually, it should be able to transform evidence into practice. In Iran, research priorities are clear to some extent; however, their active dissemination and finding solutions to assure they are performed require special interventions. Also, it is of high importance to build capacity for research in the country. Effective research requires transparent and accountable methods for budget allocation. Furthermore, the execution of research needs a knowledge network consisting of relevant institutes and research centers at national level. Nevertheless, the most important success factor of such a research system is employing capable, keen, and highly motivated researchers who have effective internal and external communications. However, our resources for achieving health targets are limited and optimizing these limited resources is of high priority. To avoid trial and error, the best evidence should be utilized to inform policy and decision making.

\section{Conflict of Interests}

The authors declare that they have no competing interests.
Strengthening evidence-based decision-making: is it possible without improving health system stewardship? Health Policy Plan. 2012;27(6):499-504.

5. Parisa Mansoori P, Majdzadeh R, Abdi Z, Rudan I, Chan K. Setting Research Priorities to Improve Population Health in Iran. J Glob Health. 2018;8(2):020702.

6. Abdi Z, Majdzadeh R, Ahmadnezhad E. Developing a framework for the monitoring and evaluation of the Health Transformation Plan in the Islamic Republic of Iran: lessons learned. East Mediterr Health J. 2018;24.

7. Yazdizadeh B, Mohtasham F. Assessment of research systems in universal health coverage-related organizations. Med J Islam Repub Iran. 2018;32(1):83-88.

8. Sajadi HS, Ehsani E, Majdzadeh R. Universal Health Coverage in Iran; where we stand and how we can move. Med J Islam Repub Iran. 2019;33(1):46-51.

\section{References}

1. World health report 2013: Research for universal health coverage.

2. Dye C, Reeder JC, Terry RF. Research for Universal Health Coverage. Sci Transl Med. 2013;5(199):199ed13-ed13.

3. Yazdizadeh B, Majdzadeh R, Janani L, Mohtasham F, Nikooee S, Mousavi A, et al. An assessment of health research impact in Iran. Health Res Policy Syst. 2016 Jul 26;14(1):56.

4. Majdzadeh R, Yazdizadeh B, Nedjat S, Gholami J, Ahghari S. 